\title{
Codeine induces increased resistance at the esophagogastric junction but has no effect on motility and bolus flow in the pharynx and upper esophageal sphincter in healthy volunteers: A randomized, double-blind, placebo-controlled, cross-over trial
}

\author{
Annelies Geeraerts $^{1}$ | Hannelore Geysen ${ }^{1}$ | Lisa Ballet ${ }^{2}$ | Claudia Hofmans $^{2}$ | \\ Egbert Clevers $^{1}$ (i) | Taher Omari ${ }^{3}$ (i) | Anastassios C. Manolakis ${ }^{4}$ | Raf Mols ${ }^{6}$ \\ Patrick Augustijns $^{6}$ | Tim Vanuytse ${ }^{1,2}$ | Nathalie Rommel ${ }^{1,2,5}$ | Jan Tack ${ }^{1,2}$ (i) | \\ Ans Pauwels ${ }^{1}$
}

${ }^{1}$ Translational Research Center for Gastrointestinal Disorders, KU Leuven, Leuven, Belgium

${ }^{2}$ Department of Gastroenterology, Neurogastroenterology and Motility, University Hospitals, Leuven, Belgium

${ }^{3}$ College of Medicine \& Public Health and Centre for Neuroscience, Flinders University, Adelaide, SA, Australia

${ }^{4}$ Department of Gastroenterology, Evangelismos General Hospital of Athens, Athens, Greece

${ }^{5}$ ExpORL Department of Neurosciences, Deglutology, KU Leuven, Leuven, Belgium

${ }^{6}$ Department of Pharmaceutical and Pharmacological Sciences, Drug Delivery and Disposition, KU Leuven, Leuven, Belgium

\section{Correspondence}

Jan Tack, Translational Research Center for Gastrointestinal Disorders, Herestraat 49, box 701, 3000 Leuven, Belgium. Email: jan.tack@med.kuleuven.be

Funding information

Ans Pauwels is supported by a postdoctoral fellowship grant from the Research Foundation Flanders (FWO Vlaanderen). Tim Vanuytsel is a senior clinical investigator of the Research Foundation Flanders (FWO Vlaanderen). Jan Tack is supported by a Methusalem grant from the KULeuven. Taher Omari holds a National Health

\begin{abstract}
Background: Chronic opioid use can induce esophageal dysfunction with symptoms resembling achalasia and a manometric pattern of esophagogastric junction-outflow obstruction (EGJ-OO). However, the effect of opioids in acute setting on pharyngeal function and esophageal body contractility has not been investigated.

Methods: After positioning the high-resolution impedance manometry (HRiM) catheter, codeine $(60 \mathrm{mg}$ ) or placebo (glucose syrup) was infused intragastrically. Fortyfive minutes post-infusion, participants received liquid, semi-solid, and solid boluses to assess esophageal and pharyngeal function. HRiM analysis was performed adhering to the Chicago classification v3.0. (CC v3.0). Pressure flow analysis (PFA) for the esophageal body and the pharynx was performed using the SwallowGateway ${ }^{\mathrm{TM}}$ online platform.
\end{abstract}

Key Results: Nineteen healthy volunteers (HV) [5 male; age 38.3] were included. After codeine administration, higher integrated relaxation pressure $4 \mathrm{~s}$ values resulted in significantly reduced deglutitive EGJ relaxation and distal latency was significantly shorter. Distal contractility was similar in both conditions. Bolus flow resistance at the EGJ and distention pressures increased significantly after codeine infusion. Based on CC v3.0, acute infusion of codeine induced EGJ-OO in six HV ( $p=0.0003$ vs. placebo). Codeine administration induced no significant alterations in any of the pharyngeal PFA metrics. 
\& Medical Research Council Senior Research Fellowship. The development of the swallowgateway.com website was supported by grants from the College of Medicine and Public Health, Flinders University. dose of codeine did not affect motility or bolus flow in pharynx and UES. ClinicalTrials. gov number, NCT03784105.

\section{KEYWORDS}

acute setting, Codeine, contractile activity and pressure flow parameters, esophageal body, pharynx

\section{1 | INTRODUCTION}

Opioids are one of the most commonly prescribed classes of drugs when it comes to pain management in modern Western society with 259 million related prescriptions in the United States in 2012. ${ }^{1,2}$ Furthermore, a study from the United Kingdom revealed a high level of non-prescription analgesics use (45\%), for example, cough syrup with codeine, in the general population. ${ }^{3}$ Opioids act through binding on the $\mu, \delta$, and $\kappa$ opioid receptors, mediating neurotransmitter release. ${ }^{4}$ The different receptor subtypes are localized in the gastrointestinal (GI) tract, although their relative distribution can vary across the different organs and tissue layers. $\mathrm{Mu}(\mu)$-receptors are the most important mediators for opioid analgesic effects and are abundantly present in the central nervous system $\left(\mu_{1}\right)$ and in the enteric nervous system of the GI-tract $\left(\mu_{2}\right){ }^{5}$ When opioids bind to these receptors, release of the neurotransmitter is suppressed, resulting in a decreased neuronal excitability. ${ }^{4}$ As $\mathrm{Gl}$ motility is regulated by neurotransmitters, opioid administration will result in abnormal coordination of motility, decreased fluid secretions, and increased sphincter tone. ${ }^{4,5}$ Possible consequences are delayed gastric, small bowel, or colon transit time, which in case of chronic use or overconsumption can lead to symptomatic gastroparesis and slow-transit constipation. ${ }^{5}$

Less is known about the effects of opioids on the esophagus. Previous research observed that chronic opioid use also affected esophageal function, causing symptoms of dysphagia and a manometric pattern of functional esophagogastric junction-outflow obstruction (EGJ-OO) and achalasia assessed by high-resolution manometry (HRM). ${ }^{6-8}$ More recently, Ortiz et al postulated a new entity known as opioid-induced esophageal dysfunction (OIED) referring to esophageal motility disorders caused by chronic use of opioids. ${ }^{9}$ It was also observed that the prevalence of OIED is higher in patients taking higher doses of opioids. ${ }^{10}$ More recently, the same research group reported that chronic opioid use affects deglutitive inhibition of the esophageal body during the multiple rapid swallows (MRS), a provocative maneuver during HRM. ${ }^{11}$ Furthermore, little is known about the effects of opioids in the proximal region of the esophagus, the upper esophageal sphincter (UES), and the pharynx. Smiley et al noted in a case study a decreased or absent swallowing function and gag reflex as a rare, but repetitive symptom after administration of the opioid fentanyl in healthy pregnant women during labor. ${ }^{12}$ Furthermore, other authors confirmed a higher incidence of pharyngeal dysfunction in healthy volunteers (HV) after morphine and remifentanil administration. ${ }^{13,14}$ Additionally, pharyngeal resistance was increased and both the vigor of the pharyngeal contraction and

\section{Key Points}

- Acute administration of codeine increased bolus resistance at the EGJ secondary to induced incomplete EGJ relaxation.

- Acute administration of codeine resultated in major motility disorders in a subset of subjects including EGJ-OO.

- Acute administration of codeine did not affect motility or bolus flow in pharynx and UES.

the pharyngeal propulsion of the bolus was decreased after administration of remifentanil, which can be linked to an increased pulmonary aspiration risk. ${ }^{14,15}$ Opioids also induced shortening of the duration of the UES opening and increased the residual UES pressure. Additionally, a reduced duration of bolus flow and increased hypo-pharyngeal distention pressure was observed after administration of remifentanil using high-resolution impedance manometry (HRiM). ${ }^{16}$

HRiM measures contractile activity of the pharynx, UES, esophageal body, and the lower esophageal sphincter (LES) during swallowing combined with bolus flow based on impedance values. ${ }^{17,18}$ Pressure flow analysis (PFA) metrics combine the analysis of contractile activity and bolus flow to describe the impact of pharyngeal and esophageal contractility on bolus transit. ${ }^{17,18}$

The effect of opioids on the pharynx, UES, and esophageal body has been studied mainly in retrospective and non-placebo-controlled settings of chronic and often high-dose opioid use. However, opioids are also widely used in acute settings and as non-prescription medications. Therefore, the aim of this study is to investigate the effect of an acute single dose of a non-prescription opioid on the contractile activity and the pressure flow parameters of the pharynx and esophageal body, assessed through HRM and PFA in a randomized, double-blinded, placebo-controlled, cross-over study.

\section{2 | MATERIALS AND METHODS}

\subsection{Subjects}

We performed this study in $\mathrm{HV}$, aged between 18 and 60 years, after obtaining written informed consent. Exclusion criteria included any chronic disease or medication intake (except oral contraception), 
chronic Gl symptoms, history of head/neck/esophageal surgery, pregnancy or breast-feeding, major allergy to codeine. The study protocol has been registered at Clinicaltrials.gov (NCT03784105), was in accordance with the Declaration of Helsinki, and was approved by the Ethics Committee of the University of Leuven (approval number S60496).

\subsection{Sample size}

This study used a randomized, placebo-controlled, double-blinded, cross-over design which aimed to include $22 \mathrm{HV}$. This number of HV is based on previous studies with similar objectives, with a medium effect size and a power level of $80 \%$ at the $p<0.05$ level of significance (calculated using $\mathrm{G}^{*}$ power 3.1.9.2) ) $^{19-21}$ and taking into account $5 \%$ of poor metabolizers.

\section{3 | Study design}

All HV were scheduled for two study visits with HRiM measurement (Laborie, Medical Measurement System, Enschede, The Netherlands) with at least one-week wash-out in between. The study outline is shown in Figure 1. After an overnight fast, HV came to the endoscopy unit of the university hospital of Leuven. A solid-state HRiM catheter (Unisensor AG, Attikon, Switzerland), with 36 pressure sensors and 16 impedance segments, was introduced transnasally into the esophagus. After an adaptation period of $15 \mathrm{~min}$, a nasogastric feeding tube (Eurosteriel Medical, The Netherlands) was positioned in the proximal stomach and codeine $(30 \mathrm{ml}$ of syrup containing $2 \mathrm{mg} / \mathrm{ml}$ codeine phosphate, Bronchodine ${ }^{\circledR}$ ) or placebo (30 $\mathrm{ml}$ of glucose syrup) was administered directly into the stomach to avoid an effect of the different taste. Thereafter, the nasogastric feeding tube was instantly removed. The $60 \mathrm{mg}$ dose of codeine was selected as it represents the maximum single dose in clinical practice. Afterward, HV were placed in a semi-recumbent position for the entire duration of the experiment.

The order of codeine and placebo administration was randomized by an online randomization tool (http://www.randomization. $\mathrm{com} /$ ) and the preparation and administration of codeine or placebo was performed by an experienced independent researcher.

Esophageal function was assessed by HRiM 45 min after the study drug administration, based on the reported peak plasma codeine levels after 60-90 min after oral ingestion. Subjects were given 10 wet swallows of $5 \mathrm{ml}$ (International Dysphagia Diet Standardisation Initiative (IDDSI) 0 or liquid boluses), followed by 5 MRS (IDDSI 0), 5 semi-solid boluses of 2 different consistencies (IDDSI 2 (thin semi-solids) and 4 (thick semi-solids)), using standardized conductivity bolus media for HRiM (SBMkit-Precise Thick'n, Trisco Food Co) and 5 solid boluses (bread $2 \times 2 \mathrm{~cm}$ ). Bread boluses contained $1 \%$ saline to enhance conductivity for impedance measurements. Approximately 30 min later, the catheter was retracted until the UES and pharyngeal functional landmarks were adequately depicted on the HRiM tracing. The pharyngeal phase was assessed using the same boluses as in the esophageal phase. After each swallow, HV were asked to fill out perception scores to describe subjective impression of the bolus transport (normal, annoying, slow, stepwise, painful, obstructive). Blood samples were taken using heparinized vacutainer tubes at 60,90, and 120 min after codeine or placebo administration to determine the levels of codeine and its demethylated metabolite morphine in plasma. Plasma was prepared by centrifuging within $30 \mathrm{~min}$ and stored at $-80^{\circ} \mathrm{C}$ until analysis.

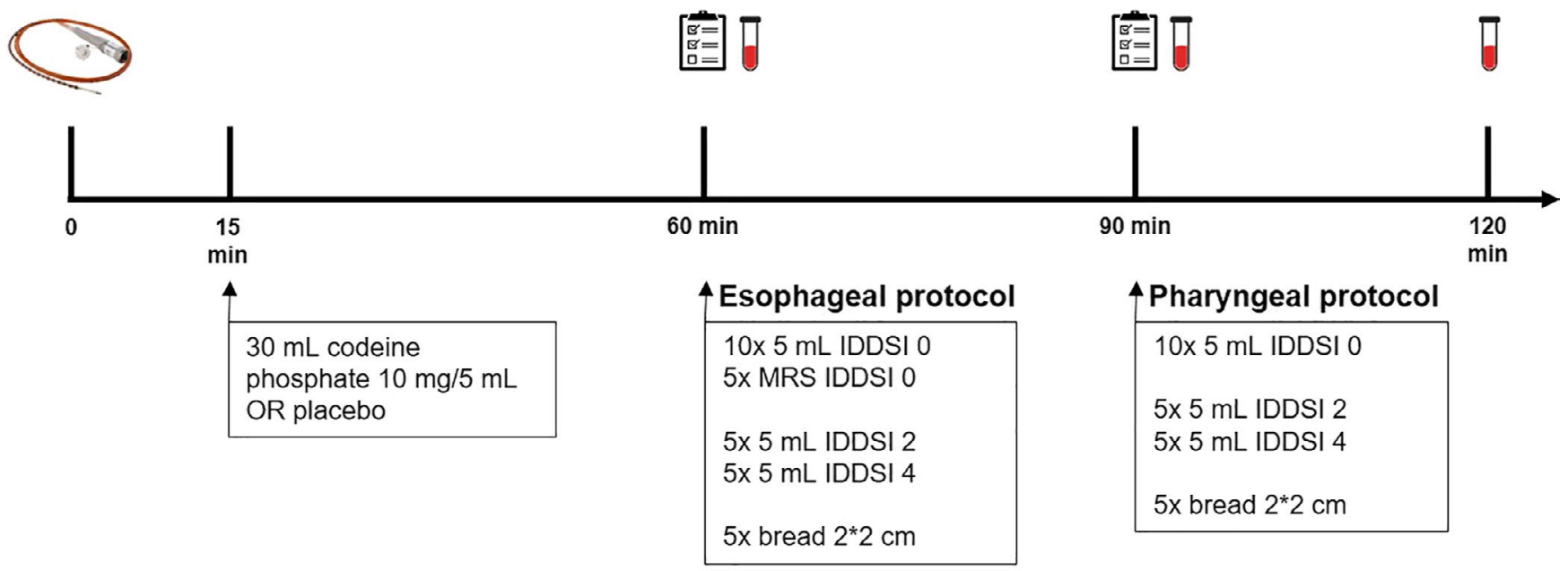

Blood sample to detect slow morphine metabolizers

$\left[\begin{array}{l}\mathbb{E}= \\ \mathbb{E}= \\ \square=\end{array}\right]$ Perception scores after each swallow

FIGURE 1 Outline of the study. Abbreviations: IDDSI, International Dysphagia Diet Standardization Initiative; MRS, Multiple Rapid Swallows 


\subsection{Poor metabolizers}

The plasma codeine-morphine ratio was measured by reverse-phase high-performance liquid chromatography with mass spectrometry detection (Acquity H-class UPLC, Waters, and Xevo TQ-S micro Waters). Separation was performed using a Kinetex XB-C18 column ( $2.6 \mu \mathrm{m}, 2.1 \times 50 \mathrm{~mm}$; Phenomenex, Utrecht, the Netherlands) held at $35^{\circ} \mathrm{C}$. Methanol (solvent A) and $0.5 \%$ formic acid in water (solvent B) were used as eluents at $500 \mu \mathrm{l} / \mathrm{min}$. Gradient elution was performed as follows: $5 \%$ of solvent $A$ during $0.3 \mathrm{~min}$, followed by $20 \% \mathrm{~A}$ for $0.8 \mathrm{~min}$. After $1.1 \mathrm{~min}$, solvent A increased from $20 \%$ to $95 \%$ to rinse the column for $1.5 \mathrm{~min}$. Prior to the next injection, the column was reequilibrated with $5 \%$ of solvent $A$ and $95 \%$ of solvent $B$ during 1.9 min. Morphine and codeine eluted after 0.59 and $1.32 \mathrm{~min}$, respectively. ${ }^{22}$

Codeine function as a prodrug, through metabolization to morphine. ${ }^{23}$ Participants were defined as slow morphine metabolizers (poor metabolizers) by a plasma concentration of morphine $<1 \mathrm{nM}$ or below detection limit and/or when the ratio of the concentration of morphine (in nM) over codeine (in nM) was $<1 \% .{ }^{24}$

\subsection{Data analysis}

First, HRM parameters were obtained for different bolus types ( $5 \mathrm{ml}$ IDDSI 0, $5 \mathrm{ml}$ IDDSI 2, $5 \mathrm{ml}$ IDDSI 4 and bread $2 \times 2 \mathrm{~cm}$ ): integrated relaxation pressure $4 \mathrm{~s}$ (IRP4), distal contractile integral (DCI) and distal latency (DL) (Table 1). These parameters were also used to determine a diagnostic category according to the Chicago classification v3.0. ${ }^{25}$ Next, DCl values were obtained for the first 4 swallows of the MRS IDDSI 0 to determine whether there was a complete or impaired deglutitive inhibition, the latter defined as 1 out of the $5 \mathrm{MRS}$ with a $\mathrm{DCl}>100 \mathrm{mmHg}^{*} \mathrm{~cm}^{*}$ s (Figure 4B). Manometric and impedance data of each trial swallow were exported from the MMS system to an ASCII file and uploaded onto the Swallow Gateway platform ${ }^{\mathrm{TM}}$ (accessed via swallowgateway. com-Flinders Partners Ptv Ltd, Flinders University, Level 2, 1284 South Road, Clovelly Park SA 5042, Australia). This online software platform was used to perform PFA and to derive biomechanical data of swallowing function based on the HRiM recordings ( $\mathrm{mmHg}$ ). To perform PFA, specific landmarks were indicated on

\begin{tabular}{|c|c|c|}
\hline Parameter & Unit & Description \\
\hline \multicolumn{3}{|l|}{ Esophageal body } \\
\hline IRP4 & $\mathrm{mmHg}$ & EGJ relaxation \\
\hline $\mathrm{DCl}$ & $\mathrm{mmHg}^{*} \mathrm{~cm}^{*} \mathrm{~s}$ & $\begin{array}{l}\text { Esophageal body contractility (strength of } \\
\text { contraction in the esophageal body) }\end{array}$ \\
\hline $\mathrm{DL}$ & s & $\begin{array}{l}\text { Time between onset of swallow (UES relaxation) } \\
\text { and the contraction deceleration point }\end{array}$ \\
\hline PFI & - & $\begin{array}{l}\text { Resistance to bolus flow at the EGJ (defined by } \\
\text { the relationship between peristaltic strength } \\
\text { and flow resistance) }\end{array}$ \\
\hline DPE & $\mathrm{mmHg}$ & Flow resistance above the EGJ \\
\hline DRP & $\mathrm{mmHg} / \mathrm{s}$ & $\begin{array}{l}\text { Flow resistance during luminal closure in distal } \\
\text { esophagus (Intrabolus pressure slope) }\end{array}$ \\
\hline $\mathrm{DCL}$ & s & $\begin{array}{l}\text { Time between maximum esophageal distension to } \\
\text { luminal closure (Time from nadir impedance to } \\
\text { peak pressure) }\end{array}$ \\
\hline \multicolumn{3}{|l|}{ Pharynx and UES } \\
\hline SRI & - & Marker of global swallow dysfunction \\
\hline UES Adm & $\mathrm{mS}$ & $\begin{array}{l}\text { Admittance in UES during deglutition reflects the } \\
\text { extent of UES opening }\end{array}$ \\
\hline IBP & $\mathrm{mmHg}$ & $\begin{array}{l}\text { Marker of resistance to bolus flow during } \\
\text { pharyngeal passage of the bolus }\end{array}$ \\
\hline VTI & $\mathrm{mmHg}^{*} \mathrm{~s}^{*} \mathrm{~cm}$ & $\begin{array}{l}\text { Marker of pharyngeal contractility from } \\
\text { velopharynx to tongue base }\end{array}$ \\
\hline PeakP & $\mathrm{mmHg}$ & Highest pharyngeal pressure in hypopharynx \\
\hline $\begin{array}{l}\text { UES post relaxation } \\
\text { PeakP }\end{array}$ & $\mathrm{mmHg}$ & Highest UES pressure post-UES relaxation \\
\hline
\end{tabular}

TABLE 1 Description of parameters used in this study

Abbreviations: $\mathrm{DCl}$, distal contractile integral; $\mathrm{DCL}$, distension-contraction latency; $\mathrm{DL}$, distal latency; DPE, distension pressure emptying; DRP, distal ramp pressure; EGJ, esophagogastric junction; IBP, intrabolus pressure; IRP4, integrated relaxation pressure $4 \mathrm{~s}$; PeakP, peak pressure; PFI, pressure flow index; SRI, swallow risk index; UES Adm, upper esophageal sphincter admittance; UES, upper esophageal sphincter; VTI, velopharyngeal to tongue basal integral. 
TABLE 2 Median values (interquartile range) for standard HRM parameters

\begin{tabular}{|c|c|c|c|c|}
\hline Consistency & Parameter & Placebo & Codeine & $p$-value \\
\hline \multirow[t]{3}{*}{$5 \mathrm{ml}$ IDDSI 0} & IRP4 (mmHg) & $10(8-14)$ & $18(15-21)$ & 0.0002 \\
\hline & $\mathrm{DCl}\left(\mathrm{mmHg}^{*} \mathrm{~cm}^{*} \mathrm{~s}\right)$ & 1431 (648-1908) & $1622(1012-2215)$ & 0.78 \\
\hline & $\mathrm{DL}(\mathrm{s})$ & $7.1(6.4-8.0)$ & $6.0(5.6-6.2)$ & $<0.0001$ \\
\hline \multirow[t]{3}{*}{$5 \mathrm{ml}$ IDDSI 2} & IRP4 (mmHg) & $9(7-17)$ & $20(17-24)$ & $<0.0001$ \\
\hline & $\mathrm{DCl}\left(\mathrm{mmHg}^{*} \mathrm{~cm}^{*} \mathrm{~s}\right)$ & 1198 (769-1828) & 1363 (886-2010) & 0.91 \\
\hline & $\mathrm{DL}(\mathrm{s})$ & $7.5(6.6-8.3)$ & $6.4(6-6.9)$ & $<0.0001$ \\
\hline \multirow[t]{3}{*}{$5 \mathrm{ml} \mathrm{IDDSI} 4$} & IRP4 (mmHg) & $12(7-17)$ & $24(18-28)$ & $<0.0001$ \\
\hline & $\mathrm{DCl}\left(\mathrm{mmHg}^{*} \mathrm{~cm}^{*} \mathrm{~s}\right)$ & 1116 (661-1726) & 1367 (820-1707) & 0.68 \\
\hline & $\mathrm{DL}(\mathrm{s})$ & $8.1(7.4-8.8)$ & $6.8(6.2-7.2)$ & $<0.0001$ \\
\hline \multirow[t]{3}{*}{ Bread $2 \times 2 \mathrm{~cm}$} & IRP4 (mmHg) & $15(11-20)$ & $26(19-36)$ & 0.0002 \\
\hline & $\mathrm{DCl}\left(\mathrm{mmHg}^{*} \mathrm{~cm}^{*} \mathrm{~s}\right)$ & 1954 (877-3106) & 1941 (1298-2712) & 0.99 \\
\hline & $\mathrm{DL}(\mathrm{s})$ & $8.2(6.9-8.8)$ & $6.4(5.7-6.9)$ & $<0.0001$ \\
\hline MRS IDDSI 0 & $\mathrm{DCl}\left(\mathrm{mmHg}^{*} \mathrm{~cm}^{*} \mathrm{~s}\right)$ & $3.5(0-34.5)$ & $151(39-551)$ & 0.008 \\
\hline
\end{tabular}

Note: $p$-values presented after stepdown Bonferroni correction.

Abbreviations: $\mathrm{DCl}$, distal contractile integral; DL, distal latency; IDDSI, international dysphagia diet standardization initiative; IRP, integrated relaxation pressure $4 \mathrm{~s}$; MRS, multiple rapid swallow. the uploaded Clouse plot and following parameters were calculated for the esophageal body (Table 1): distension pressure emptying (DPE), distal ramp pressure (DRP), and distension-contraction latency (DCL). The following PFA metrics were calculated for the pharynx and UES (Table 1): swallow risk index (SRI), UES admittance (UES Adm), intrabolus pressure (IBP), velopharyngeal to tongue basal integral (VTI), peak pressure (PeakP) in hypopharynx and UES post relaxation peak pressure (UES post relaxation PeakP).

PFA parameters were obtained for the same bolus characteristics as the HRM parameters ( $5 \mathrm{ml} \mathrm{IDDSI} \mathrm{0/2/4} \mathrm{and} \mathrm{bread} 2 \times 2 \mathrm{~cm}$ ). Median values for each PFA parameter per consistency was calculated.

\subsection{Statistical analysis}

Poor metabolizers and subjects with major motility disorders according to Chicago criteria v3.0 during the placebo condition were excluded from the analysis. Data were analyzed using SAS University Edition (SAS Institute). Mixed models were constructed with the metrics outlined in Table 1 as the dependent variable and bolus consistency (IDDSI 0/2/4 and solids) and treatment condition (codeine or placebo) as within-subject independent, categorical variables. If necessary, data were converted using a logarithmic transformation or BoxCox transformation to fulfill the assumption of normally distributed residuals in mixed model analysis. Effects of interest included a main effect of condition to observe an effect of the codeine administration on the defined parameters over the different consistencies. In case a significant main effect of the treatment was observed, post hoc analyses were performed of each consistency individually to detect a difference in the defined parameters, after the codeine administration. Correction for multiple testing was applied, using stepdown Bonferroni. The Chicago criteria-based diagnoses and the proportion of impaired deglutitive inhibition during MRS in both conditions were compared using McNemar testing. Differences in perception scores were compared between test conditions using the nonparametric paired Wilcoxon signed-rank test. Data are presented as median (interquartile range) unless specified otherwise. Significance level was set at a $p$-value $<0.05$.

\section{3 | RESULTS}

\section{1 | Subjects}

Twenty-seven HV were included in the study. Eight participants were excluded for the analysis: one due to biliary type pain, a rare side effect of codeine, which led to preterm end of the experiment, one due to the presence of an EGJ-OO during the placebo condition and six were poor metabolizers ( 3 men). In total, $19 \mathrm{HV}$ (5 male; age $38 \pm 3$ years; body mass index $\left(23.5 \pm 0.6 \mathrm{~kg} / \mathrm{m}^{2}\right)$ were included in the final analysis. For the analysis of the deglutitive inhibition of the MRS, the data of only $18 \mathrm{HV}$ was included, due to missing MRS data from one participant.

\section{2 | Esophageal body}

Median (interquartile range) values for the studied HRM parameters (IRP4, DCl, and DL) of the different bolus characteristics are presented in Table 2

After codeine administration, significantly higher values for IRP4 were observed ( $p<0.0001$ ). Post hoc analysis revealed a significant difference in IRP4 values compared with placebo for all bolus types (liquids, semi-solids, and solids) (Table 2 and Figure 2A). 
(A)

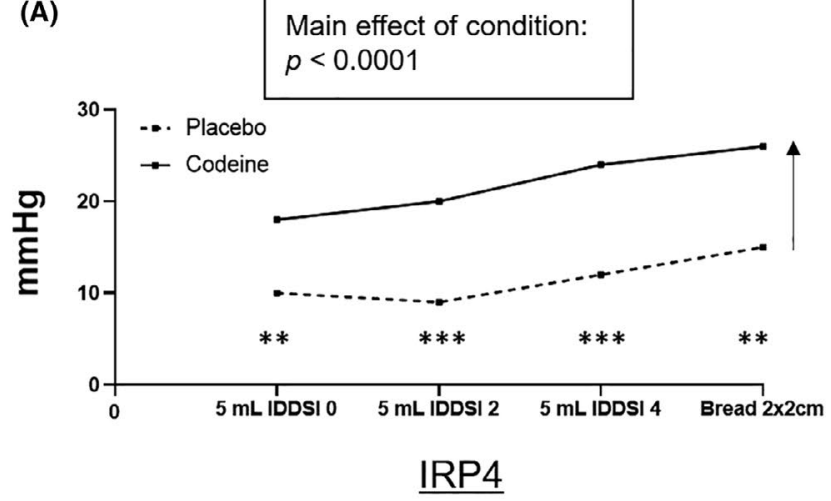

(B)

\section{No main effect of} condition

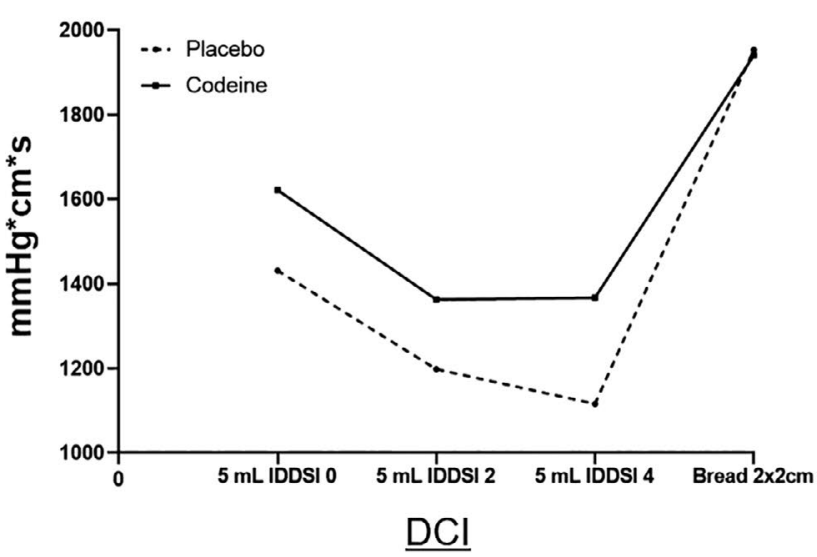

(C)

Main effect of condition: $p<0.0001$

FIGURE 2 The effect of codeine on IRP4, DCI, and DL values (presented as median). $p$-values after post hoc testing: ${ }^{*} p<0.05$, ${ }^{* *} p<0.01$, and ${ }^{* * *} p<0.001$. Abbreviations: DCI, distal contractile integral; DL, distal latency; HRM, high-resolution manometry; IDDSI, international dysphagia diet standardization initiative; IRP4, integrated relaxation pressure $4 \mathrm{~s}$

TABLE 3 Perception scores measured after every swallow during codeine administration and placebo for the esophageal body and pharynx

\begin{tabular}{|lccccc} 
& \multicolumn{2}{l}{ Esophageal body } & & \multicolumn{2}{l}{ Pharynx } \\
\cline { 2 - 3 } \cline { 6 - 6 } \cline { 5 - 6 } Normal & Codeine & Placebo & & Codeine & Placebo \\
\hline Annoying & $1.7 \%$ & $1.9 \%$ & & $0.0 \%$ & $0.8 \%$ \\
\hline Stepwise & $1.1 \%$ & $0.4 \%$ & & $0.0 \%$ & $1.1 \%$ \\
\hline Slow & $7.4 \%$ & $1.1 \%$ & & $6.7 \%$ & $3.4 \%$ \\
\hline Painful & $0.0 \%$ & $0.0 \%$ & & $0.0 \%$ & $0.0 \%$ \\
\hline Obstructive & $0.2 \%$ & $1.3 \%$ & & $0.2 \%$ & $1.7 \%$ \\
\hline
\end{tabular}

Note: Data presented as percentages for the total number of swallows (liquids (IDDSI 0), semi-solids (IDDSI 2 and 4), and solids (bread).

Abbreviation: IDDSI, international dysphagia diet standardization initiative.

Although $\mathrm{DCl}$ values were numerically higher after codeine infusion for the boluses of $5 \mathrm{mI}$ IDDSI $0 / 2 / 4$, this did not reach the level of statistical significance (Table 2 and Figure 2B). Lower values for DL were observed after codeine infusion $(p<0.0001)$. Post hoc analysis demonstrated that for all bolus consistencies, DL was significantly shortened after the codeine administration (Table 2 and Figure 2C).

After each swallow, participants were asked to rate their perception score. After placebo and codeine administration no significant differences were observed, with the overall majority of the swallows being perceived as normal (95.4\% and $89.7 \% ; p=0.11$; Table 3).

Based on the Chicago Classification v3.0, acute administration of codeine induced an EGJ-OO, major esophageal motility disorder, in six HV ( $p=0.0003$; Figure 3).

After codeine administration, significantly higher $\mathrm{DCl}$ values were observed during the MRS ( $p=0.008$ ) (Table 2). Furthermore, codeine significantly induced impaired deglutitive esophageal inhibition in a larger proportion of the HV compared to placebo $(p=0.02$; Figure 4).

The esophageal PFA parameters (PFI, DPE, DRP, and DCL) are shown in Table 4. In general, after codeine infusion, significantly higher values for PFI were observed $(p=0.03)$ and post hoc tests confirmed statistical significance for all bolus types (liquids, 
(A)
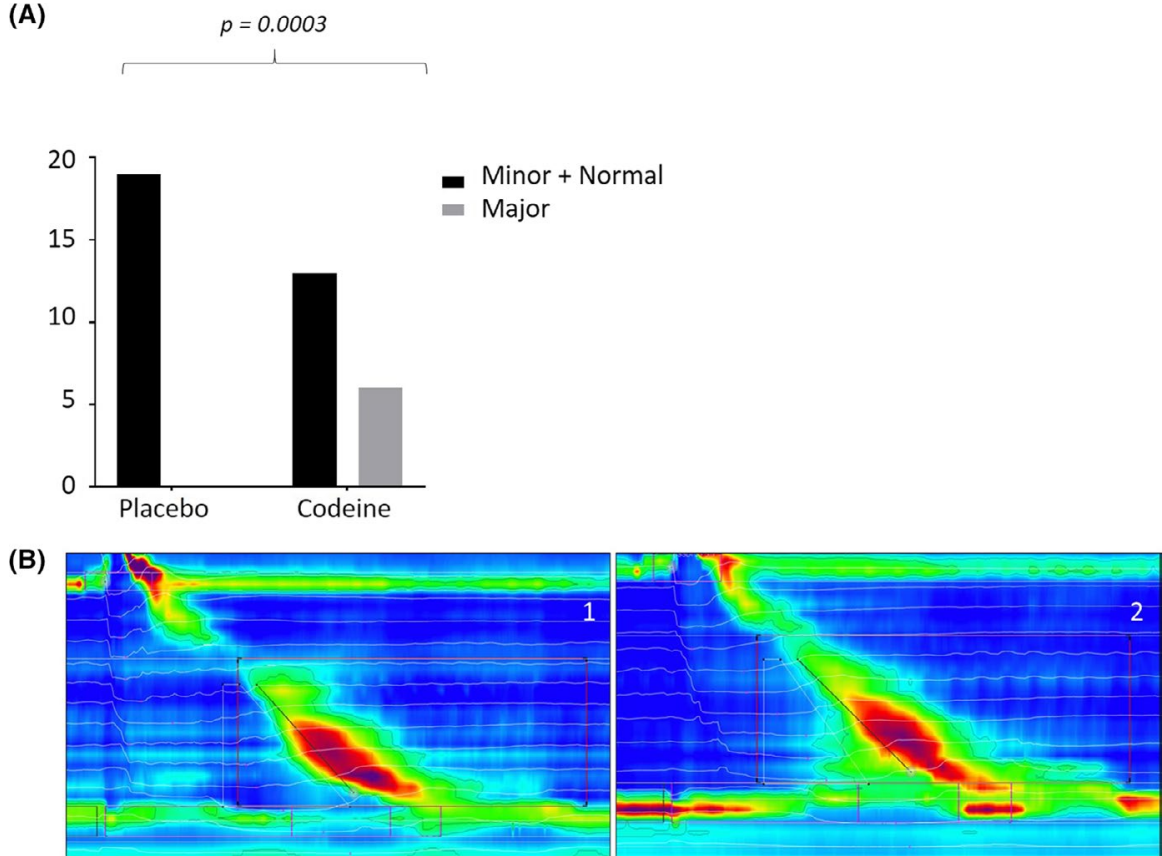

FIGURE 3 Change induced by acute codeine administration. A, Change (in absolute numbers) for esophageal motility disorders assessed by the Chicago classification v3.0 before and after codeine administration. B, HRiM read-out from one healthy volunteer who developed an EGJ-OO after acute codeine administration. (1) After placebo administration: IRP4 = $12 \mathrm{mmHg} ; \mathrm{DCl}=1473 \mathrm{mmHg}{ }^{*} \mathrm{~cm}^{*}$; $; \mathrm{DL}=7.4 \mathrm{~s}$ : normal. (2) After one single maximum dose of codeine administration: IRP4 $=30 \mathrm{mmHg}$; DCl $=2006 \mathrm{mmHg}^{*} \mathrm{~cm} * \mathrm{~s}$; DL = $7.1 \mathrm{~s}$ : EGJ-OO (major motility disorder). Abbreviations: DCl, distal contractile integral; DL, distal latency; EGJ-OO, esophagogastric junction-outflow obstruction; HRiM, high-resolution impedance manometry; IRP4, integrated relaxation pressure $4 \mathrm{~s}$

(A)

\begin{tabular}{|l|l|l|l|}
\hline Deglutitive inhibition & Placebo & Codeine & P-value \\
\hline Complete & 11 & 7 & \multirow{2}{*}{0.02} \\
\hline Impaired & 4 & 14 & \\
\hline
\end{tabular}

(B)
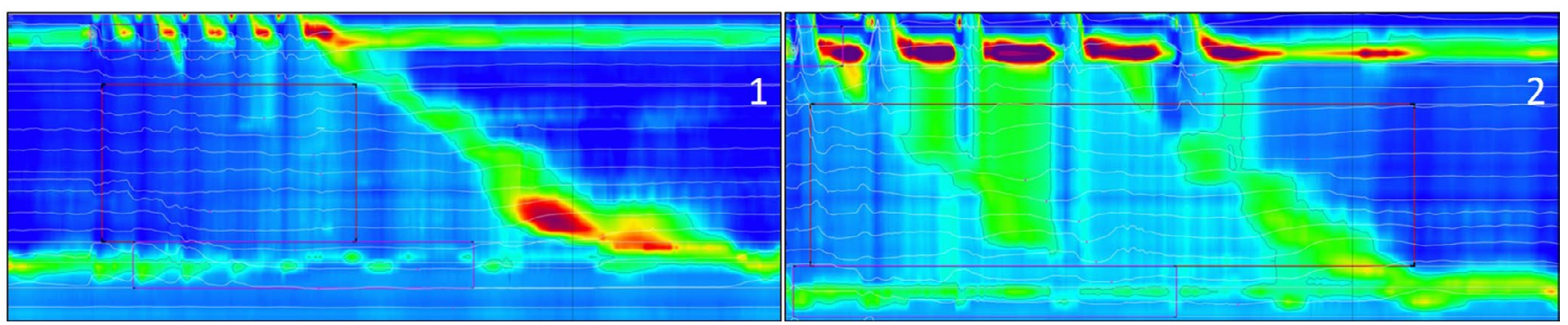

FIGURE 4 Number of complete versus impaired deglutitive esophageal inhibition induced by acute codeine administration during MRS. A, Number of HVs with complete versus impaired deglutitive esophageal inhibition during MRS during placebo and codeine condition. B, HRiM read-out from one healthy volunteer who evolved from complete to impaired deglutitive esophageal inhibition during MRS after acute codeine administration. (1) After placebo administration: $\mathrm{DCl}=2 \mathrm{mmHg}^{*} \mathrm{~cm}^{*} \mathrm{~s}$. (2) After one single maximum dose of codeine administration: $\mathrm{DCl}=1987 \mathrm{mmHg}^{*} \mathrm{~cm}^{*}$ s. Abbreviations: $\mathrm{DCl}$, distal contractile integral; HRiM, high-resolution impedance manometry; MRS, multiple rapid swallows

semi-solids, and solids) (Table 4 and Figure 5A). Higher values for DPE were observed after codeine treatment ( $p=0.0005)$, with a significant effect for all bolus types (Table 4 and Figure 5B). No significant differences were observed for DRP $(p=0.08)$ (Table 4 and
Figure 5C). Finally, DCL values were significant decreased after codeine intake $(p=0.002$ ). Post hoc analysis revealed statistical significance for the liquid (IDDSI 0) and thick semi-solids (IDDSI 4) boluses only (Table 4 and Figure 5D). 


\begin{tabular}{|c|c|c|c|c|}
\hline Consistency & Parameter & Placebo & Codeine & $p$-value \\
\hline \multirow[t]{4}{*}{$5 \mathrm{ml} \mathrm{IDDSI} 0$} & $\mathrm{PFI}$ & $11.8(8.9-24.4)$ & $41.8(26.6-70.8)$ & 0.003 \\
\hline & $\mathrm{DPE}(\mathrm{mmHg})$ & $11.6(10.3-15.10)$ & $16.4(13.2-19.2)$ & 0.03 \\
\hline & $\mathrm{DRP}(\mathrm{mmHg} / \mathrm{s})$ & $3.2(1.8-5)$ & $4.8(3.8-6.9)$ & 0.28 \\
\hline & $\mathrm{DCL}(\mathrm{s})$ & $3.3(2.4-4.3)$ & $2.7(2.2-2.9)$ & 0.005 \\
\hline \multirow[t]{4}{*}{$5 \mathrm{ml}$ IDDSI 2} & $\mathrm{PFI}$ & $27.4(16.6-46.5)$ & $66.1(40.4-188.8)$ & 0.09 \\
\hline & $\mathrm{DPE}(\mathrm{mmHg})$ & $15.1(11.8-16.7)$ & $19.7(15.6-27.9)$ & 0.03 \\
\hline & $\mathrm{DRP}(\mathrm{mmHg} / \mathrm{s})$ & $4.5(3.2-7.4)$ & $6.5(4.9-12.4)$ & 0.47 \\
\hline & $\mathrm{DCL}(\mathrm{s})$ & $2.3(1.9-2.9)$ & $1.9(1.5-2.5)$ & 0.13 \\
\hline \multirow[t]{4}{*}{$5 \mathrm{ml} \mathrm{IDDSI} 4$} & $\mathrm{PFI}$ & $81.4(36.2-137.4)$ & $181.2(94.3-545.8)$ & 0.010 \\
\hline & $\mathrm{DPE}(\mathrm{mmHg})$ & $16.8(13.1-20.1)$ & $27.8(22.4-35)$ & $<0.0001$ \\
\hline & $\mathrm{DRP}(\mathrm{mmHg} / \mathrm{s})$ & $7.1(3.4-10.2)$ & $7.4(4.1-14.6)$ & 0.47 \\
\hline & $\mathrm{DCL}(\mathrm{s})$ & $1.8(1.6-2.2)$ & $1.5(1.2-1.6)$ & 0.002 \\
\hline \multirow[t]{4}{*}{ Bread $2 \times 2 \mathrm{~cm}$} & PFI & $113.2(34.4-449.1)$ & $\begin{array}{l}338.2 \\
(191.9-622.6)\end{array}$ & 0.25 \\
\hline & $\mathrm{DPE}(\mathrm{mmHg})$ & $18.9(14.3-23.4)$ & $34.2(24.2-44.3)$ & $<0.0001$ \\
\hline & $\mathrm{DRP}(\mathrm{mmHg} / \mathrm{s})$ & $8.8(3.5-23.2)$ & $12.2(6.9-20.6)$ & 0.47 \\
\hline & $\mathrm{DCL}(\mathrm{s})$ & $1.6(1.4-2.1)$ & $1.3(0.9-1.8)$ & 0.09 \\
\hline
\end{tabular}

TABLE 4 Median values (interquartile range) for esophageal pressure flow analysis

Note: $p$-values presented after stepdown Bonferroni correction.

Abbreviations: DCL, distension-contraction latency; DPE, distension pressure emptying; DRP, distal ramp pressure; IDDSI, international dysphagia diet standardization initiative; PFI, pressure flow index.

\section{3 | Pharynx and UES}

There were no significant differences in any of the pharyngeal PFA parameters between the codeine and placebo condition (Table 5). Increased bolus consistency also increased the UES admittance, representing deglutitive UES opening, in both the placebo and the codeine condition (main effect of consistency, $p<0.001$ ). Increasing the bolus consistency also resulted in increased UES post relaxation PeakP (main effect of consistency, $p=0.007$ ). Post hoc analysis only revealed a significantly higher UES post relaxation PeakP in the solid bolus (bread $2 \times 2 \mathrm{~cm}$ ) compared to the liquid bolus (IDDSI 0 ) $(p=0.02)$. However, no interaction effect between condition and bolus consistency was observed, both for UES Adm ( $p=0.86)$ as UES post relaxation PeakP ( $p=0.91)$.

Both in the placebo and codeine condition, the perception of the swallows was rated as normal in $93.1 \%$ of the swallows (Table 3 ).

\section{4 | DISCUSSION}

Opioids are among the most commonly prescribed drug classes for pain management in the Western world and even the use of nonprescription analgesics is very high in the general population. ${ }^{1-3}$ The aim of our study was to investigate the effect of an acute single dose of a non-prescription opioid on the contractile activity and the pressure flow parameters of the pharynx and esophageal body.

In the esophageal body, this study showed significantly higher values for IRP4 and decreased values for DL after acute administration with a maximum dosage of codeine. Numerically higher values for $\mathrm{DCl}$ were observed, but they did not reach statistical significance. This result could be due to a type II error (ß), although with a power level $(1-\beta)$ of $78 \%$, the probability of avoiding this type of error is kept as low as possible. In 6 out of $19 \mathrm{HV}$ the administration of codeine resulted in a manometric pattern of an EGJ-OO, a major esophageal motility disorder classified according the Chicago Classification v3.0, but without induction of dysphagia. EGJ-OO is characterized by IRP4 levels higher than the upper limit of normal (cut off = IRP4 $\geq 20 \mathrm{mmHg}$ ) with at least some preservation of peristalsis. ${ }^{25}$ The DL was significantly decreased without reaching the manometric cut off of $<4.5 \mathrm{~s}$ to diagnose esophageal spasm.

Already in 1983, Rattan and Goyal, demonstrated 5 opioid receptor subtypes in the LES. ${ }^{26}$ Several years later, other researchers suggested that LES relaxation is mediated through activation of inhibitory neurons in the myenteric plexus by opioid receptors (both $\mu$ and $\delta$ ) regulating relaxation. ${ }^{27}$ Previous research concerning chronic opioid use already confirmed the effect on LES functioning, causing symptoms similar to achalasia and a manometric pattern of functional EGJ-OO or achalasia in some patients. ${ }^{6,7}$ The results of this study showed that already a single, acute administration, of a non-prescription opioid, is able to increase EGJ resistance in $\mathrm{HV}$ and is able to mimic a major motility disorder such as an EGJ-OO in a subset of subjects.

This study is the first, to the best of our knowledge, to confirm the development of an EGJ-OO and impaired deglutitive inhibition after a single maximum dose of codeine administration. This study supports the theory by Snyder et al. that opioids impair inhibitory 
(A)

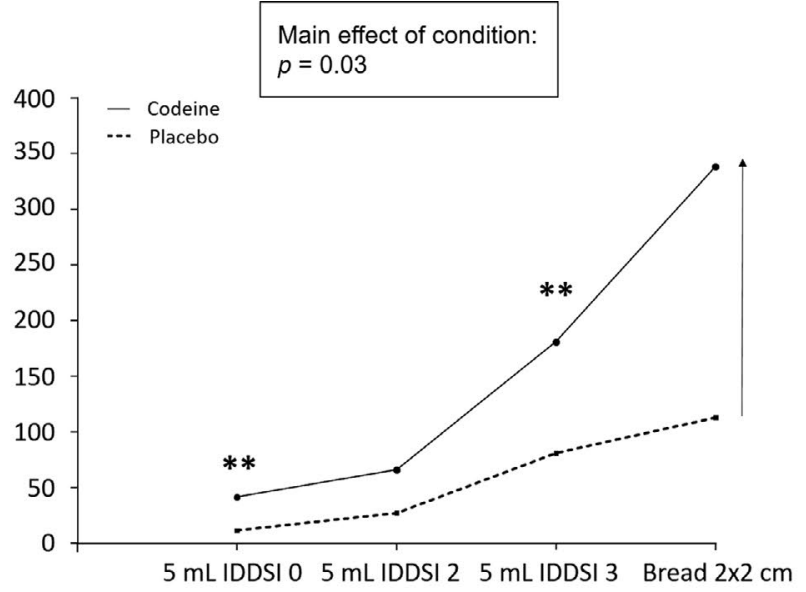

$\underline{P F I}$

(C)

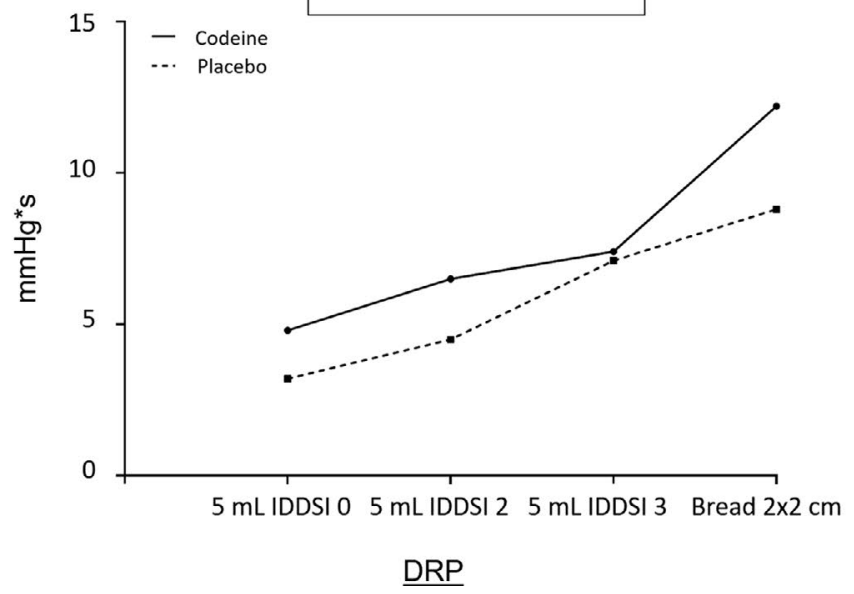

(B)

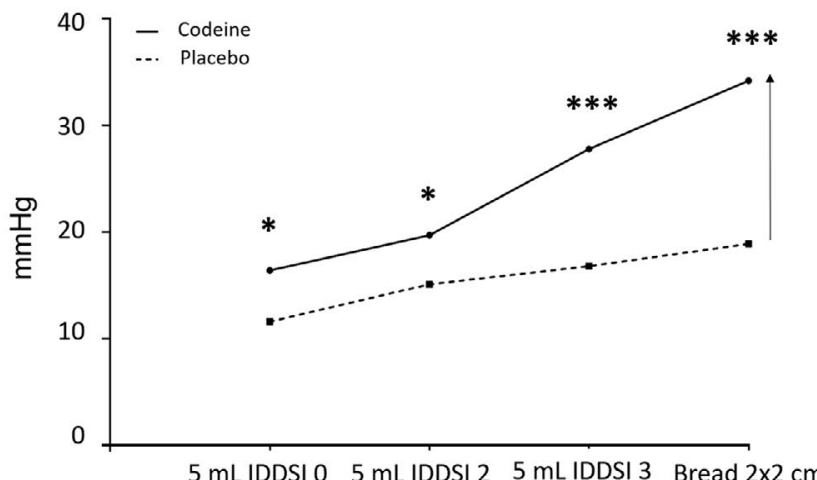

DPE

(D)

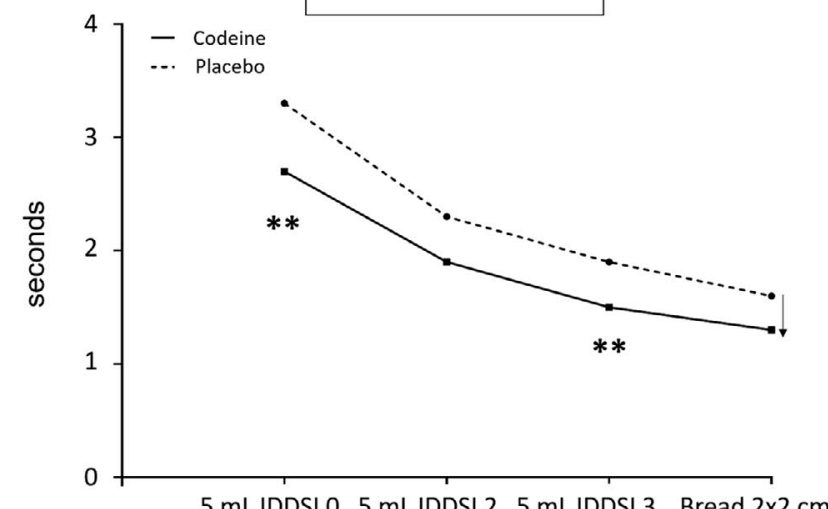

$\underline{\mathrm{DCL}}$

FIGURE 5 Effect of codeine on PFA parameters before and after codeine administration. A, Significant increase of PFI values after codeine administration. B, Significant increase of DPE values after codeine administration. C, No significant increase of DRP after codeine administration, although a numerically difference was observed. D, Significant decrease in DCL values after codeine administration. $p$-values after post hoc testing: ${ }^{*} p<0.05,{ }^{* *} p<0.01$, and ${ }^{* * *} p<0.001$ ). Abbreviations: $\mathrm{DCL}$, distension-contraction latency; DPE: distension pressure emptying; DRP, distal ramp pressure; IDDSI, International Dysphagia Diet Standardization Initiative; PFA, pressure flow analysis; PFI, pressure flow index

neural pathways which results in abnormal contractile activity during MRS. ${ }^{11}$ Additionally, this study was the first to unravel the effect of a non-prescription opioid on the esophagus using PFA, which combines contractile activity and bolus flow. After codeine administration, we observed higher values of $\mathrm{PFI}$, indicating a higher resistance to bolus flow at the EGJ. Next, codeine administration increased DPE values and decreased DCL values, which results in a higher resistance at the EGJ level and a faster contraction of the esophagus after maximum distension. Finally, despite evidence of augmented flow resistance induced by codeine, this was insufficient to produce a significant increase in the bolus pressure ramp observed during luminal closure (DRP). Overall, the PFA revealed an increased bolus flow resistance at the EGJ in HV after codeine administration, which is in line with the demonstrated effects of codeine on the HRM parameters and the development of an EGJ-OO.
This study demonstrated that an acute single dose codeine had no significant effect on the pharyngeal motility and bolus flow. Moreover, codeine had no significant effect on the UES opening, nor did it induce a higher resistance at the level of the pharynx and UES. This is in contrast with other studies in the literature. Smiley and Moore noticed a loss of swallowing ability after administration of fentanyl which was reversed by naloxone ${ }^{12}$ and Savilampi et $\mathrm{al}^{28}$ reported an increase in SRI with remifentanil and morphine while in our study the SRI parameter was not significantly affected by acute codeine administration. These contrasting findings could be explained by differences in the study protocols. Smiley and Moore based their conclusion on the observation that two women reported to have swallowing difficulties after the administration of subarachnoid fentanyl. ${ }^{12}$ However, these observations were not based on objective measurements, whereas our 


\begin{tabular}{|c|c|c|c|c|}
\hline Consistency & Parameter & Placebo & Codeine & $p$-value \\
\hline \multirow[t]{6}{*}{$5 \mathrm{ml} \mathrm{IDDSI} 0$} & SRI & $1.2(0.2-1.9)$ & $0.7(0.3-1.5)$ & 0.99 \\
\hline & UES Adm (mS) & $3.4(3.1-3.9)$ & $3.4(3.1-3.7)$ & 0.54 \\
\hline & IBP $(\mathrm{mmHg})$ & $7.9(0.3-9.0)$ & $4.8(1.5-10.3)$ & 0.69 \\
\hline & VTI (mmHg*s*cm) & $132.3(94.1-170.5)$ & $\begin{array}{l}144.2 \\
(102.9-190.9)\end{array}$ & 0.42 \\
\hline & PeakP (mmHg) & $\begin{array}{l}188.0 \\
(166.3-266.4)\end{array}$ & $\begin{array}{l}191.2 \\
(153.1-234.7)\end{array}$ & 0.59 \\
\hline & $\begin{array}{c}\text { UES post relaxation } \\
\text { PeakP }(\mathrm{mmHg})\end{array}$ & $\begin{array}{l}277.2 \\
(202.1-369.1)\end{array}$ & $\begin{array}{l}264.4 \\
(198.5-318.4)\end{array}$ & 0.31 \\
\hline \multirow[t]{6}{*}{$5 \mathrm{ml} \mathrm{IDDSI} 2$} & SRI & $0.7(0.2-1.8)$ & $0.7(0.3-1.3)$ & 0.68 \\
\hline & UES Adm (mS) & $4.0(3.4-4.1)$ & $3.8(3.7-4.1)$ & 0.85 \\
\hline & $\mathrm{IBP}(\mathrm{mmHg})$ & $4.1(2.0-7.4)$ & $3.8(2.0-9.5)$ & 0.48 \\
\hline & VTI (mmHg $\left.{ }^{*} \mathrm{~s}^{*} \mathrm{~cm}\right)$ & $128.4(94.8-152.2)$ & $132.7(90.4-194.0)$ & 0.28 \\
\hline & PeakP $(\mathrm{mmHg})$ & $\begin{array}{l}195.9 \\
(154.5-232.4)\end{array}$ & $\begin{array}{l}184.6 \\
(143.8-224.6)\end{array}$ & 0.19 \\
\hline & $\begin{array}{c}\text { UES post relaxation } \\
\text { PeakP }(\mathrm{mmHg})\end{array}$ & $\begin{array}{l}268.4 \\
(200.0-386.6)\end{array}$ & $\begin{array}{l}263.6 \\
(206.0-328.7)\end{array}$ & 0.43 \\
\hline \multirow[t]{6}{*}{$5 \mathrm{ml} \mathrm{IDDSI} 4$} & SRI & $0.9(0.3-1.9)$ & $1.3(0.4-1.9)$ & 0.93 \\
\hline & UES Adm (mS) & $4.1(3.9-4.2)$ & $4.2(4.0-4.4)$ & 0.94 \\
\hline & IBP $(\mathrm{mmHg})$ & $6.8(0.7-11.8)$ & $8.8(3.2-14.8)$ & 0.57 \\
\hline & VTI (mmHg $\left.{ }^{*}{ }^{*} \mathrm{~cm}\right)$ & $\begin{array}{l}142.5 \\
(105.0-172.9)\end{array}$ & $118.7(83.1-203.6)$ & 0.97 \\
\hline & PeakP (mmHg) & $\begin{array}{l}200.6 \\
(141.5-270.2)\end{array}$ & $\begin{array}{l}183.1 \\
(142.3-240.8)\end{array}$ & 0.17 \\
\hline & $\begin{array}{c}\text { UES post relaxation } \\
\text { PeakP }(\mathrm{mmHg})\end{array}$ & $\begin{array}{l}289.7 \\
(214.0-366.2)\end{array}$ & $\begin{array}{l}286.4 \\
(205.9-349.9)\end{array}$ & 0.22 \\
\hline \multirow{6}{*}{$\begin{array}{l}\text { Bread } \\
\qquad 2 \times 2 \mathrm{~cm}\end{array}$} & SRI & $0.4(0.2-1.1)$ & $0.4(0.2-0.7)$ & 0.21 \\
\hline & UES Adm (mS) & $3.4(3.0-3.6)$ & $3.2(2.8-3.6)$ & 0.41 \\
\hline & $\mathrm{IBP}(\mathrm{mmHg})$ & $3.7(0.8-11.0)$ & $3.1(0.3-7.4)$ & 0.09 \\
\hline & VTI (mmHg $\left.{ }^{*}{ }^{*} \mathrm{~cm}\right)$ & $\begin{array}{l}159.1 \\
(121.5-179.4)\end{array}$ & $\begin{array}{l}172.3 \\
(115.1-206.9)\end{array}$ & 0.84 \\
\hline & PeakP (mmHg) & $\begin{array}{l}198.6 \\
(168.6-239.4)\end{array}$ & $\begin{array}{l}194.1 \\
(156.9-242.3)\end{array}$ & 0.40 \\
\hline & $\begin{array}{c}\text { UES post relaxation } \\
\text { PeakP }(\mathrm{mmHg})\end{array}$ & $\begin{array}{l}278.7 \\
(220.6-435.3)\end{array}$ & $\begin{array}{l}318.0 \\
(220.4-384.9)\end{array}$ & 0.67 \\
\hline
\end{tabular}

TABLE 5 Median values (interquartile range) for liquids (IDDSI 0), semi-solids (IDDSI 2 and 4) and solids (bread) after placebo and codeine administration

Note: $p$-values presented after stepdown Bonferroni correction.

Abbreviations: IBP, intrabolus pressure; IDDSI, international dysphagia diet standardization initiative; PeakP, peak pressure; SRI, swallow risk index; UES Adm, upper esophageal sphincter admittance; VTI, velopharyngeal to tongue basal integral.

findings were based on objective parameters obtained from HRiM measurements. Savilampi et al ${ }^{28}$ included a group of participants above 65 years old, who could already had some subtle pre-existing swallow dysfunction, which may be exacerbated by the administration of opioids. Additionally, a different dose and mode of drug administration were used. Salivampi et $\mathrm{al}^{28}$ administered the remifentanil and morphine via an IV infusion and injection respectively, whereas in our study the codeine was administered intragastrically.

Furthermore, our negative findings regarding the pharynx and UES may be explained by a less pronounced effect of opioids on striated muscle function, in contrast to their effects on the enteric nervous system in the distal esophagus with smooth muscle cell layers. However, Salivampi et al did report a difference in SRI after administration of remifentanil and morphine in $\mathrm{HV}^{15,28}$ These contrasting findings could be explained by differences in the study protocols as explained above. Nonetheless, $\mu$-receptor opioids have also been detected in striated muscle in rats, ${ }^{29}$ but data on human pharynx and UES is still lacking.

A limitation of this study was that we did not include administration of naloxone at the end of the study protocol, to reverse the effect of codeine. This could give additional confirmation that the observed effect is mediated through $\mu$-receptors, as codeine and naloxone have the highest affinity for this subtype of opioid 
receptor. ${ }^{30-32}$ Furthermore, in this study we investigated the effect of one single acute dose of codeine and these results cannot be generalized for chronic codeine intake.

Furthermore, 24\%, 6 out of 25, were found to be poor metabolizers which was higher than the $5 \%$ as we expected to observe. ${ }^{33}$ Due this higher number, we were not able to perform the analysis with $22 \mathrm{HV}$ (with 5\% poor metabolizers), which was the proposed sample size based on our initial power calculation. However, based on a post hoc power calculation, with a medium effect size, $p<0.05$ level of significance, and $19 \mathrm{HV}$ we reached a power level of $78 \%$, which is a very small and probably negligible difference with the $80 \%$ level.

In conclusion, acute codeine administration, a non-prescription opioid, increased EGJ resistance in HV and induced a manometric pattern of a major motility disorder such as an EGJ-OO in a subset of subjects. Next, acute codeine administration resulted in impaired deglutitive inhibition during MRS. Furthermore, this was supported by the analysis of PFA parameters, which showed increased bolus resistance at the EGJ in $\mathrm{HV}$ after acute codeine administration. Therefore, precaution is needed when performing and interpreting HRiM measurements in patients treated with opioids. Acute administration of codeine had no influence on the pharynx nor on the UES. Further analysis is needed to explain the mechanisms underlying this phenomenon.

\section{ACKNOWLEDGMENT}

The authors are very grateful to L. Timmermans for ordering the test meals and all her help during this study.

\section{DISCLOSURE}

Jan Tack has given Scientific advice to AlfaWassermann, Allergan, Christian Hansen, Danone, Grünenthal, Ironwood, Janssen, Kiowa Kirin, Menarini, Mylan, Neutec, Novartis, Noventure, Nutricia, Shionogi, Shire, Takeda, Theravance, Tramedico, Truvion, Tsumura, Zealand and Zeria pharmaceuticals, has received research support from Shire, Sofar and Tsumura, and has served on the Speaker bureau for Abbott, Allergan, AstraZeneca, Janssen, Kyowa Kirin, Menarini, Mylan, Novartis, Shire, Takeda, Truvion and Zeria. Nathalie Rommel and Taher Omari hold a patent on the AIM software used for pressure-flow analysis. The other authors have no other conflicts of interest to declare.

\section{AUTHOR CONTRIBUTION}

ACM, NR, TV, JT and AP were responsible for the study concept and design. AG, HG, $L B, C H$, and $A P$ were involved in the acquisition of data. Data analysis was performed by AG, HG, LB, CH, EC and NR. Interpretation of data was performed by AG, HG, LB, CH, TV, NR, JT and AP. AG, HG, NR, TV, JT and AP were responsible for the draft of the manuscript. Codeine analysis were performed by RM and PA. TO provided Swallow Gateway SMBkit ${ }^{\text {TM }}$. All authors were involved in the critical revision of the manuscript for important intellectual content and all authors reviewed and approved the final version of the manuscript.

\section{ORCID}

Egbert Clevers (D) https://orcid.org/0000-0003-1931-4926

Taher Omari (D) https://orcid.org/0000-0001-5108-7378

Jan Tack (D) https://orcid.org/0000-0002-3206-6704

\section{REFERENCES}

1. Serpell M. Handbook of Pain Management. London: Current Medicine Group; 2008.

2. Lacy BE. Effects of opioids on esophageal dysfunction. Gastroenterology \& hepatology. 2016;12:323-325.

3. Porteous T, Bond C, Hannaford P, et al. How and why are non-prescription analgesics used in Scotland? Fam Pract. 2005;22:78-85.

4. Wood JD, Galligan JJ. Function of opioids in the enteric nervous system. Neurogastroenterol Motil. 2004;16(Suppl 2):17-28.

5. Brock C, Olesen SS, Olesen AE, et al. Opioid-induced bowel dysfunction: pathophysiology and management. Drugs. 2012;72:1847-1865.

6. Gonzalez ES, Bellver VO, Jaime FC, et al. Opioid-induced lower esophageal sphincter dysfunction. I Neurogastroenterol Motil. 2015;21:618-620.

7. Ratuapli SK, Crowell MD, DiBaise JK, et al. Opioid-induced esophageal dysfunction (OIED) in patients on chronic opioids. Am J Gastroenterol. 2015;110:979-984.

8. Cock C, Doeltgen SH, Omari T, et al. Effects of remifentanil on esophageal and esophagogastric junction (EGJ) bolus transit in healthy volunteers using novel pressure-flow analysis. Neurogastroenterol Motil. 2018;30(2):e13191. https://doi.org/10.1111/nmo.13191

9. Ortiz V, Garcia-Campos M, Saez-Gonzalez E, et al. A concise review of opioid-induced esophageal dysfunction: is this a new clinical entity? Dis Esophagus. 2018;31:1-6.

10. Snyder DL, Crowell MD, Horsley-Silva J, et al. Opioid-induced esophageal dysfunction: differential effects of type and dose. Am J Gastroenterol. 2019;114:1464-1469.

11. Snyder DL, Valdovinos LR, Horsley-Silva J, et al. Opioids interfere with deglutitive inhibition assessed by response to multiple rapid swallows during high-resolution esophageal manometry. Am J Gastroenterol. 2020;115:1125-1128.

12. Smiley RM, Moore RP. Loss of gag reflex and swallowing ability after administration of intrathecal fentanyl. Anesthesiology. 2007;106:1253.

13. Hardemark Cedborg Al, Sundman E, Boden K, et al. Effects of morphine and midazolam on pharyngeal function, airway protection, and coordination of breathing and swallowing in healthy adults. Anesthesiology. 2015;122:1253-1267.

14. Savilampi J, Magnuson A, Ahlstrand R. Effects of remifentanil on esophageal motility: a double-blind, randomized, cross-over study in healthy volunteers. Acta Anaesthesiol Scand. 2015;59:1126-1136.

15. Savilampi J, Ahlstrand R, Magnuson A, et al. Aspiration induced by remifentanil: a double-blind, randomized, crossover study in healthy volunteers. Anesthesiology. 2014;121:52-58.

16. Doeltgen SH, Omari TI, Savilampi J. Remifentanil alters sensory neuromodulation of swallowing in healthy volunteers: quantification by a novel pressure-impedance analysis. Am J Physiol Gastrointest Liver Physiol. 2016;310:G1176-G1182.

17. Patel DAK, Robert T, Vaezi MF. Evaluation and Management of Dysphagia. Berlin, Germany: Springer.

18. Ferris $L$, Omari $T$, Selleslagh $M$, et al. Pressure flow analysis in the assessment of preswallow pharyngeal bolus presence in Dysphagia. Int J Otolaryngol. 2015;2015:764709.

19. Penagini BB, Negri G, et al. Effect of loperamide on lower oesophageal sphincter pressure in idiopathic achalasia. Scand J Gastroenterol. 1994;29:1057-1060. 
20. Penagini R, Picone A, Bianchi PA. Effect of morphine and naloxone on motor response of the human esophagus to swallowing and distension. Am J Physiol. 1996;271:G675-G680.

21. Scarpellini E, Pauwels A, Vos R, et al. Effect of methylnaltrexone and naloxone on esophageal motor function in man. Neurogastroenterol Motil. 2017;29:1-7.

22. Goelen N, de Hoon J, Morales JF, et al. Codeine delays gastric emptying through inhibition of gastric motility as assessed with a novel diagnostic intragastric balloon catheter. Neurogastroenterol Motil. 2020;32:e13733.

23. Smith HS. Opioid metabolism. Mayo Clin Proc. 2009;84:613-624.

24. Kirchheiner J, Schmidt H, Tzvetkov M, et al. Pharmacokinetics of codeine and its metabolite morphine in ultra-rapid metabolizers due to CYP2D6 duplication. Pharmacogenomics J. 2007;7:257-265.

25. Kahrilas PJ, Bredenoord AJ, Fox M, et al. The Chicago Classification of esophageal motility disorders, v3.0. Neurogastroenterol Motil. 2015;27:160-174.

26. Rattan S, Goyal RK. Identification and localization of opioid receptors in the opossum lower esophageal sphincter. J Pharmacol Exp Ther. 1983;224:391-397.

27. Barnette MS, Grous M, Manning CD, et al. Inhibition of neuronally induced relaxation of canine lower esophageal sphincter by opioid peptides. Eur J Pharmacol. 1990;182:363-368.

28. Savilampi J, Omari T, Magnuson A, et al. Effects of remifentanil on pharyngeal swallowing: a double blind randomised cross-over study in healthy volunteers. Eur J Anaesthesiol. 2016;33:622-630.
29. Storr M, Geisler F, Neuhuber WL, et al. Endomorphin-1 and -2, endogenous ligands for the mu-opioid receptor, inhibit striated and smooth muscle contraction in the rat oesophagus. Neurogastroenterol Motil. 2000;12:441-448.

30. Sawynok J, Pinsky C, LaBella FS. On the specificity of naloxone as an opiate antagonist. Life Sci. 1979;25:1621-1632.

31. Paterson SJ, Robson LE, Kosterlitz HW. Classification of opioid receptors. Br Med Bull. 1983;39:31-36.

32. Bradley CM, Nicholson AN. Effects of a mu-opioid receptor agonist (codeine phosphate) on visuo-motor coordination and dynamic visual acuity in man. Br J Clin Pharmacol. 1986;22:507-512.

33. Vieira CMP, Fragoso RM, Pereira D, et al. Pain polymorphisms and opioids: an evidence based review. Mol Med Rep. 2019;19:1423-1434.

How to cite this article: Geeraerts A, Geysen H, Ballet L, et al. Codeine induces increased resistance at the esophagogastric junction but has no effect on motility and bolus flow in the pharynx and upper esophageal sphincter in healthy volunteers: A randomized, double-blind, placebocontrolled, cross-over trial. Neurogastroenterology \& Motility. 2020;00:e14041. https://doi.org/10.1111/nmo.14041 